\title{
Eficiencia de selección indirecta en maíz para ambiente futuro por cambio climático
}

\author{
Efficiency of indirect selection in maize for future environment by climate change \\ Ramírez-Mandujano, C. A. ${ }^{\circledR}$ González-Cortés, J. C. ${ }^{1}$, Ávila-Bautista A. ${ }^{1}$, Hernández-Esquivel, A. A. ${ }^{1}$ \\ ${ }^{1}$ Facultad de Biología, Universidad Michoacana de San Nicolás de Hidalgo. Edificio R, Ciudad \\ Universitaria, Morelia, Michoacán; México. Tel. 01 (443) 316-7412 \\ Autor para correspondencia: cramirzm@umich.mx
}

Recibido: 04/01/2016

Aceptado: 10/06/2016

\begin{abstract}
RESUMEN
En la Mesa Central Mexicana se siembran en marzo y abril, más de 800 mil ha de maíz con humedad residual y más de 650 mil bajo secano en junio. Se puede esperar que por cambio climático desaparecerá la humedad residual y toda la superficie tendrá que ser sembrada bajo secano. Existen muchos trabajos de mejoramiento genético de maíz en humedad residual, y nos podemos preguntar si será útil el avance por selección actualmente alcanzado al verse forzados a retrasar la fecha de siembra. Se evaluaron 40 familias de medios hermanos de una población criolla mejorada en ambos ambientes. Se midieron días a floración masculina y femenina, asincronía floral, largo y ancho basal de la hoja de la mazorca principal, número de hojas sobre y debajo de la mazorca, altura de mazorca, altura total de planta y peso de mazorca en 8 plantas de cada familia y cada repetición. Se estimó heredabilidad, correlación genética, respuesta a selección directa en ambiente de humedad residual e indirecta en secano. La correlación genética entre ambos ambientes fue positiva para todas las variables, excepto peso de mazorca. Los valores de heredabilidad fueron mayores en la siembra de humedad residual para días a floración masculina y femenina, número de hojas, altura de planta y de mazorca, e inferiores para el resto, excepto asincronía. La selección en humedad residual provocará en cambios en el mismo sentido al mover a la población al ambiente de secano, excepto para peso de mazorca.
\end{abstract}

Palabras clave: Zea mays, cambio climático, respuesta a selección indirecta.

\begin{abstract}
In the Central Mexican Plateau are planted in March and April, more than 800 thousand ha of maize with residual moisture and more than 650 thousand ones under rainfed condition in June. We can predict that residual moisture will disappear by climate change and the entire surface will need to be planted under rainfed. There are many works on maize breeding in residual moisture, and we can ask ourselves if the currently reached advancement by selection will be useful when being forced to postpone the date of sowing. 40 families of half SIBS of a Creole improved population in both
\end{abstract}


environments were evaluated. Was measured days to masculine and femenine flowering and anthesis-silking interval, long and basal width of the leaf of the main ear, number of leaves above and below the ear, cob height, plant height and ear weight in 8 plants of every family and every repetition. We estimated heritability, genetic correlation, response to direct selection in residual humidity and indirect selection in rainfed environment. Genetic correlation between both environments was positive for all variables, except ear weight. Heritability values were higher in the residual moisture environment for days to masculine and femenine flowering, number of leaves, plant and ear height, and lower for the rest, except floral asynchrony. Selection in residual moisture will cause changes in the same direction when move the population to the rainfed environment, except for ear weight.

Keywords: Zea mays, climate change, indirect selection response.

\section{INTRODUCCIÓN}

El maíz es el cultivo agrícola más importante de México, tanto desde el punto de vista alimentario, como industrial, político y social. De esta especie, se cosecharon en 2014 cerca de 23 millones de $\mathrm{t}$ en 7 millones de ha (SIAP, 2015). En la Mesa Central de México se siembran antes del inicio de las lluvias, en los meses de marzo y abril, más de $800 \mathrm{mil}$ ha con maíz tardío bajo régimen de humedad residual y otras más de 650 mil bajo secano ("temporal") al inicio de las lluvias en el mes de junio, con maíz precoz. El rendimiento promedio bajo humedad residual es $83 \%$ superior al de secano (Arellano et al., 2011); Sin embargo se puede esperar que a futuro, por el cambio climático la disminución de la precipitación (Sáenz-Romero et al., 2012) y el acortamiento de la estación lluviosa (Ruiz et al., 2000), provocarán la desaparición de la humedad residual y que para entonces toda la superficie tendrá que ser sembrada bajo secano, hecho ya documentado en una localidad del Estado de México (Cruz, 2010). Existen muchos trabajos de mejoramiento genético de maíz en siembras de humedad residual, y nos podemos preguntar: ¿Qué pasará con ellos cuando desaparezcan las siembras de marzo-abril y dichas poblaciones tengan que ser sembradas en junio?, ¿Será útil el avance por selección actualmente alcanzado al verse forzados a retrasar la fecha de siembra? La respuesta puede estar en la estimación del efecto de selección indirecta, es decir, ¿qué efecto tendrá la selección aplicada en el ambiente de humedad residual, en el comportamiento de la población creciendo en el ambiente de secano?

Falconer y Mackay (1996) describen la selección indirecta refiriéndose a aplicar selección en un ambiente para obtener una respuesta en otro, y hay trabajos que manejan este concepto (Bänziger et al., 1997; Atlin et al., 2001; Windhausen et al., 2012; Kebede et al., 2013). En el presente estudio el objetivo fue evaluar el efecto de la selección en una población de maíz en el ambiente de humedad residual, al llevarla al ambiente de secano.

\section{MATERIALES Y MÉTODOS}

El estudio se llevó a cabo en los ciclos primavera-verano 2013 y 2014 en la Ciénega de Zacapu, Michoacán, de clima templado subhúmedo $\mathrm{C}(\mathrm{w} 1)$, con una precipitación anual de $877 \mathrm{~mm}$ y temperatura media anual de $16.7{ }^{\circ} \mathrm{C}$ (SMN, 2012). En dicha región, la mitad del área agrícola tiene humedad residual suficiente para permitir la siembra con humedad residual en marzo-abril; la otra mitad se siembra bajo secano en junio.

Se utilizaron familias de medios hermanos maternos seleccionadas al azar en el campo de cultivo, para representar genotipos de la población criolla mejorada "Tukuru", bien adaptada a la siembra de humedad residual en la Ciénega de Zacapu. El 
diseño experimental fue bloques completos al azar con tres repeticiones. Se sembraron dos ensayos, uno bajo humedad residual y otro en secano en el año 2013, este último se perdió y fue necesario repetir la siembra en 2014. La parcela experimental fue de un surco de $5 \mathrm{~m}$ conteniendo 11 matas de dos plantas cada 50 $\mathrm{cm}$, con separación de $80 \mathrm{~cm}$ entre surcos, esto es $50 \mathrm{mil}$ plantas/ha. La dosis de fertilización fue de 100-50-00. Se tomaron datos de días a floración masculina y femenina, asincronía floral, largo y ancho basal de la hoja de la mazorca principal, número de hojas sobre y debajo de la mazorca, altura de mazorca, altura total de planta y peso de mazorca en 8 plantas de cada familia y cada repetición. La variable asincronía floral se transformó a logaritmo de base e de raíz cuadrada de Asi+10. Se estimaron componentes de varianza con el procedimiento VARCOMP opción REML con el paquete estadístico SAS (SAS 2003). Se hicieron estimaciones de varianza aditiva y heredabilidad en sentido estricto a nivel de plantas individuales ( $\left.\mathrm{h}^{2} \mathrm{i}\right)$ de acuerdo a Márquez y Sahagún (1994). Se hizo pronóstico de avance por selección masal estratificada directa en el ambiente de humedad residual como lo presentan Peña et al., (2002):

$$
\mathrm{RSME}=\mathrm{ck} \sigma^{2} \mathrm{~A} /\left(\sigma_{\mathrm{f}}^{2}+\sigma_{\mathrm{r}}^{2}{ }_{\mathrm{f}}+\sigma^{2} \mathrm{e}\right)^{1 / 2}
$$

Donde: RSME = pronóstico absoluto de avance por selección masal estratificada, $\mathrm{c}=$ control parental $=0.5, \mathrm{k}=$ presión de selección estandarizado $=2.063$ cuando se selecciona al $5 \%$ de las plantas, $\sigma^{2} \mathrm{~A}=$ varianza aditiva, $\sigma_{\mathrm{f}}^{2}=$ varianza de familias, $\sigma^{2}{ }^{2} * \mathrm{f}=$ varianza de familias por repeticiones, $\sigma^{2} \mathrm{e}=$ varianza del error.

Trabajando con datos estandarizados se estimó la respuesta a selección indirecta en el ambiente de secano de acuerdo a Falconer y Mackay (1996):

$$
\begin{gathered}
\mathrm{Ri}=0.5 * \mathrm{k} * \mathrm{hx} * \text { hy* } \mathrm{rGxy} \sigma \mathrm{py} \\
\mathrm{rGxy}=\left(\sigma^{2} \mathrm{f}(\mathrm{x}+\mathrm{y})-\left(\sigma^{2} \mathrm{fx}+\sigma^{2} \mathrm{fy}\right) / 2\right) /\left(\sigma^{2} \mathrm{fx}\right.
\end{gathered}
$$

Donde: $\mathrm{Ri}$ es la respuesta a selección indirecta, $\mathrm{k}=$ presión de selección estandarizado, hx es la raíz cuadrada de la heredabilidad para el carácter en el ambiente de humedad residual, hy es la raíz cuadrada de la heredabilidad para el mismo carácter en el ambiente de secano rGxy = correlación genética entre los caracteres $\mathrm{x} \mathrm{e} \mathrm{y} ; \sigma^{2} \mathrm{f}(\mathrm{x}+\mathrm{y})=$ varianza de familias para la suma de las variables $\mathrm{x}$ e $\mathrm{y} ; \sigma^{2} \mathrm{fx}=$ varianza de familias para la variable $\mathrm{x} ; \sigma^{2}$ fy $=$ varianza de familias para la variable $y$.

\section{RESULTADOS Y DISCUSIÓN}

Como se esperaba, la siembra de secano o estación de crecimiento reducida, tuvo valores inferiores en todas las variables medidas (Dijak et al., 1999), excepto en asincronía floral, lo que refleja una condición de estrés (Bolaños y Edmeades, 1996). La superioridad de la siembra de humedad residual es del doble para hojas debajo de la mazorca y del triple para peso de mazorca (cuadro 1).

Cuadro 1. Valores promedio para las variables evaluadas

\begin{tabular}{lcccccccccccr}
\hline & FlorM & FlorF & Asi & HA & HD & HT & Long H & Ancho H & Área F & Alt M & Alt T & Peso M \\
& & & & & & & & & & & & \\
Secano & 84.36 & 88.32 & $\mathbf{4 . 4 3}$ & 4.1 & $\mathbf{4 . 7 1}$ & 8.8 & 91.2 & 10.01 & 915.57 & 106.07 & 215.33 & $\mathbf{9 4 . 8 8}$ \\
Humedad & 98.96 & 102.08 & $\mathbf{3 . 4 5}$ & 5.4 & $\mathbf{9 . 8 2}$ & 15.22 & 105.55 & 11.36 & 1203 & 145.06 & 271.99 & $\mathbf{2 9 7 . 2 6}$ \\
\hline
\end{tabular}

FlorM = días a floración masculina, FlorF = días a floración femenina, Asi = asincronía floral en días, HA = hojas arriba de la mazorca, $\mathrm{HD}=$ hojas debajo de la mazorca, $\mathrm{HT}=$ hojas totales, Long $\mathrm{H}=$ longitud de hoja $(\mathrm{cm}), \mathrm{Ancho} \mathrm{H}=$ ancho de hoja $(\mathrm{cm})$, Área $\mathrm{F}=$ área foliar $\left(\mathrm{cm}^{2}\right)$, Alt $\mathrm{M}=$ altura de mazorca $(\mathrm{cm})$, Alt $\mathrm{T}=$ altura total de planta $(\mathrm{cm})$, Peso $\mathrm{M}=$ peso de mazorca $(\mathrm{g})$. 
Los valores de heredabilidad fueron mayores en la siembra de humedad residual respecto a la de secano para días a floración masculina y femenina, número de hojas por arriba de la mazorca, hojas por debajo de la misma, hojas totales, altura de mazorca y altura total de planta. Fueron muy inferiores para largo y ancho de hoja, área foliar y peso de mazorca, y pueden atribuirse al menos en parte a una alta uniformidad ambiental. Banziger et al., (1997) encontraron también diferencias en el valor de heredabilidad para rendimiento en trigo en ambientes de alto y bajo nivel de nitrógeno (ambiente favorable y desfavorable). Para asincronía floral no hubo diferencia (cuadro 2). Los valores corresponden a los comúnmente reportados en la bibliografía (Hallauer y Miranda, 1988; Bolaños y Edmeades, 1996; Fountain y Hallauer, 1996; Muhamad y Muhamad, 2002; Soleri y Smith, 2002; Smalley et al., 2004; Cervantes-Ortiz et al., 2007; Badu-Apraku et al., 2012). La heredabilidad del peso de mazorca en el ambiente de secano fue muy superior a la del ambiente de humedad residual, donde tuvo un valor cercano a cero; conviene citar como antecedente a Atif y Atif (2011), que reportan un valor estimado negativo para heredabilidad en rendimiento en un primer año de evaluación, y de 0.22 en un segundo año. Probablemente en una evaluación adicional se obtenga un valor más alto.

Cuadro 2. Valores estimados de heredabilidad $\left(\mathrm{h}^{2}\right)$ en sentido estricto.

\begin{tabular}{lccllllllrrrr}
\hline & FlorM & FlorF & Asi & HA & HD & HT & Long H & Ancho H & Área F & Alt M & Alt T & Peso M \\
Humedad & 1 & 0.92 & $\mathbf{0 . 3 6}$ & 0.56 & 0.60 & 0.85 & 0.02 & 0.12 & $\mathbf{0 . 0 2}$ & 0.83 & 0.60 & $\mathbf{0 . 0 3}$ \\
Secano & 0.79 & 0.53 & $\mathbf{0 . 3 4}$ & 0.44 & 0.14 & 0.31 & 0.36 & 0.45 & $\mathbf{0 . 3 1}$ & 0.48 & 0.45 & $\mathbf{0 . 4 0}$ \\
\hline
\end{tabular}

FlorM = días a floración masculina, FlorF = días a floración femenina, Asi = asincronía floral, HA = hojas arriba de la mazorca, $\mathrm{HD}=$ hojas debajo de la mazorca, $\mathrm{HT}=$ hojas totales, Long $\mathrm{H}=$ longitud de hoja, Ancho $\mathrm{H}=$ ancho de hoja, Área $\mathrm{F}=$ área foliar, Alt $\mathrm{M}=$ altura de mazorca, Alt $\mathrm{T}$ = altura total de planta, Peso $\mathrm{M}$ = peso de mazorca.

La correlación genética entre ambos ambientes fue positiva para todas las variables, excepto para peso de mazorca, pero con valor bajo (cuadro 3). Entonces la selección en ambos ambientes traerá cambios en el mismo sentido cuando la población sea llevada al otro ambiente, ocurriendo lo contrario sólo para el peso de la mazorca, que es el carácter que más impacta al productor. Excepto para rendimiento de grano, los resultados son similares a los de Kebede et al., (2013), que estudiando el comportamiento de líneas endogámicas y sus cruzas en México $y$ el este y sur de África encontraron correlaciones de 0.72 o superiores para rendimiento de grano, días a floración femenina y altura de planta, concluyendo que puede intercambiarse material genético entre ambos ambientes.

Cuadro 3. Valores de correlación genotípica ( $\mathrm{rGxy}$ ) entre ambos ambientes y pronóstico de respuesta a selección indirecta $\left(\mathrm{R}_{\mathrm{cy}}\right)$ practicada en humedad residual al trasladarse a secano, así como respuesta a selección directa (RSME) en ambiente de humedad residual, expresadas en porcentaje.

\begin{tabular}{|c|c|c|c|c|c|c|c|c|c|c|c|c|}
\hline & FlorM & FlorF & Asi & $\mathrm{HA}$ & HD & $\mathrm{HT}$ & Long $\mathrm{H}$ & Ancho H & Área F & Alt M & Alt T & Peso M \\
\hline rGxy & 0.81 & 0.90 & 0.70 & 0.79 & 0.81 & 0.85 & 1.53 & 1 & 2.15 & 0.79 & 0.64 & -0.12 \\
\hline $\mathrm{K}_{\mathrm{cy}}$ & 2.98 & 3.62 & 21.20 & $7 \cdot 30$ & 5.65 & 6.29 & 1.34 & 2.89 & 3.14 & 9.67 & 4.12 & -0.55 \\
\hline RSME & 1.92 & 2.24 & 21.33 & 21.35 & 12.5 & 11.55 & 0.05 & 2.09 & 0 & 1.17 & 0.46 & 0.02 \\
\hline
\end{tabular}

FlorM = días a floración masculina, FlorF = días a floración femenina, Asi = asincronía floral, HA = hojas arriba de la mazorca, HD = hojas debajo de la mazorca, HT = hojas totales, Long $\mathrm{H}=$ longitud de hoja, Ancho $\mathrm{H}=$ ancho de hoja, Área $\mathrm{F}=$ área foliar, Alt $\mathrm{M}=$ altura de mazorca, Alt $\mathrm{T}=$ altura total de planta, Peso $\mathrm{M}=$ peso de mazorca.

En el mismo cuadro 3 se presenta la respuesta esperada a selección directa e indirecta en porcentaje. La selección directa por días a floración masculina y femenina, 
longitud y ancho de hoja, área foliar, altura de mazorca y altura total de planta traerá cambios aún mayores en el ambiente de secano, incluso peso de mazorca, con la diferencia de que el cambio será negativo; por el contrario para número de hojas arriba y debajo de la mazorca y hojas totales se manifestará un avance menor al del ambiente de humedad. La selección por peso de mazorca en ambiente de humedad residual se traducirá en una merma de poco más de medio punto porcentual por cada ciclo de selección al llevar a la población al ambiente de secano, en parte por el muy bajo valor de heredabilidad en el ambiente de selección, pero hay que tomar en cuenta que el ambiente de secano puede considerarse un ambiente de baja productividad y al respecto Atlin et al., (2001) señalan que programas formales de mejoramiento genético han sido exitosos en desarrollar variedades de alto rendimiento llevando múltiples evaluaciones a través de ambientes favorables, pero menos exitosos en desarrollar materiales que superen a los maíces locales en ambientes marginales. Windhausen et al., (2013) coinciden en lo anterior cuando dicen que la división en sub regiones es innecesaria solo cuando hay poca diferencia entre ellas, lo que no aplica en este trabajo. También Banziger et al., (1997) concluyeron que la selección en ambiente de alto nitrógeno para comportamiento en bajo nitrógeno traerá una reducción en rendimiento cuando la deficiencia excede $43 \%$.

\section{CONCLUSIONES}

La siembra en secano muestra comportamiento inferior en todas las variables medidas, excepto asincronía floral. La superioridad de la siembra de humedad residual es el triple para peso de mazorca. La correlación genética entre ambos ambientes fue positiva para todas las variables, excepto peso de mazorca. Los valores de heredabilidad fueron mayores en la siembra de humedad residual para días a floración masculina y femenina, número de hojas, altura de planta y de mazorca. Fueron inferiores para largo y ancho de hoja, área foliar y peso de mazorca. La selección en humedad residual se manifestará en cambios en el mismo sentido al mover a la población al ambiente de secano, excepto para peso de mazorca, donde habrá una merma de $0.5 \%$ por cada ciclo de selección.

\section{LITERATURA CITADA}

Arellano-Vázquez, J.L.; Virgen-Vargas J.; Rojas-Martínez, I.; Ávila-Perches, M.A. 2011. H-70: híbrido de maíz de alto rendimiento para temporal y riego del altiplano central de México Rev. Mex. de Ciencias Agrícolas 2(4): 619-626. https://doi.org/10.29312/remexca.v2i4.1

Atif, E.I.; Atif, I.A. 2011. Genetic Variability For Vegetative and Yield Traits in Maize (Zea Mays L.) International Research Journal of Agricultural Science And Soil Science. 1(10): 408411.

Atlin, G.N.; Cooper M.; Å. Bjørnstad. 2001. A comparison of formal and participatory breeding approaches using selection theory. Euphytica 122(3): 463-475. https://doi.org/10.1023/A:101755730780 Badu-Apraku, B.; Akinwale, R.O.; Facorede, M.A.B.; Oyekunle, M.; Franco, J. 2012. Relative changes in genetic variability and correlation in an early-maturing maize population during recurrent selection. Theor appl genet (2012) 125:1289-1301 https://doi.org/10.1007/s00122-012-1913

Bänziger, M; Betrán, F. J.; Lafitte, H. R. 1997. Efficiency of High-Nitrogen Selection Environments for Improving Maize for Low-Nitrogen Target Environments. Crop Sci. 37(4): 11031109 https://doi.org/10.2135/cropsci1997.001

Bolaños, J.; Edmeades G.O. 1996. The importance of the anthesis-silking interval in breeding for drought tolerance in tropical maize. Field Crops Res.48(1):65-80. https://doi.org/10.1016/

Cervantes-Ortiz, F.; García de los Santos, G.; Carballo-Carballo, A.; Bergvinson, D.; Crossa, J.L.; Mendoza-Elos, M.; Moreno-Martínez, E. 2007. Herencia del vigor de plántula y su relación con caracteres de planta adulta en líneas endogámicas de maíz tropical. Agrociencia 41: 425-433. 92 
Cruz,M. 2010. Comparación del ciclo agrícola actual con el de hace unos diez años en San Juan Jalpa municipio San Felipe del Progreso, Estado de México: evidencia de adaptación al cambio climático. Ra Ximhai 7(1):95-106.

https://doi.org/10.35197/rx.07.01.2011.0

Dijak, M.; Modarres, A.M.; Hamilton, R.I.; Dwyer, L.M.; Stewart, D.W.; Mather, D.E.; Smith, D.L. 1999. Leafy ReducedStature Maize Hybrids for Short-Season Environments. Crop Sci. 39(4):11061110. https://doi.org/10.2135/cropsci19

Falconer, D.S.; Mackay, T.F.C. 1996. Introduction to Quantitative Genetics. 4.ed. England. Longman, 1996. $464 \mathrm{p}$.

Fountain, M.O.; Hallauer, A.R. 1996. Genetic variation within maize breeding populations. Crop Science 36 : 26-32. https://doi.org/10.2135/cropsci1996.001

Hallauer, A.R.; Miranda, J.B. 1988. Quantitative genetics in maize breeding. $2^{a}$ Ed. Iowa State University Prees. Ames, Io. 468 pp.

Kebede, A.Z.; Mahuku, G.; Burgueño, J.; San Vicente, F.; Cairns, J.E.; Das, B.; Makumbi, D.; Magorokosho, C.; Windhausen, V.S.; Melchinger, A.E.; Atlin, G.N. 2013. Effectiveness of selection at CIMMYT's main maize breeding sites in Mexico for performance at sites in Africa and vice versa. Plant Breeding 132: 299-304 https://doi.org/10.1111/pbr.12063

Márquez-Sánchez, F.; Sahagún-Castellanos J. 1994. Estimation of genetic variances with maternal half-sib families. Maydica 39: 197-201.

Muhamad, Y.; Muhamad, S. 2002. Estimates of heritability for some quantitative characters in maize. International journal of agriculture \& biology 4(1): 103-104.

Peña L.A.; Molina, G.J.D.; Márquez, S.F.; Sahagún, C.J.; Ortiz, C.J.; Cervantes, S.T. 2002. Respuestas estimadas y observadas de tres métodos de selección en tomate de cáscara (Physalis ixocarpa Brot.) Revista Fitotecnia Mexicana 25: 171-178.

Ruiz, C.A.; Ramírez, J.L.; Flores, F.J.; Sánchez J.J. 2000. Cambio climático y su impacto sobre la estación de crecimiento de maíz en Jalisco, México. Fitotecnia Mexicana 23(2): 169-181.

Sáenz-Romero, C.; Rehfeldt, G.E.; Crookston, N.L.; Duval, P.; Beaulieu, J. 2012. Spline models of contemporary, 2030, 2060 and 2090 climates for Michoacán state, México. Impacts on the vegetation. Fitotecnia Mexicana 35(4):333-345.

https://doi.org/10.35196/rfm.2012.4.333

SAS Institute. 2003. SAS version 9.1.3 for Windows. SAS Institute, Inc. Cary, NC.

Servicio Meteorológico Nacional (SMN). 2012.

http://smn.cna.gob.mx/climatologia/Est adistica/16188.pdf;

http://smn.cna.gob.mx/climatologia/Est adistica/16171.pdf. (Consultado el 16 de abril de 2012).

Sistema de Información Agroalimentaria y

Pesquera (SIAP). 2015. Anuario Agropecuario 1980-2009.

http://www.siap.gob.mx/cierre-de-laproduccion-agricola-por-cultivo/ Consulta: junio 24, 2015.

Smalley, M.D.; Daub, J.L.; Hallauer, A.R. 2004. Estimation of heritability in maize by parent - offspring regression. Maydica 49(3): 221-229.

Soleri, D.; Smith, S.E. 2002. Rapid estimation of broad sense heretability of farmermanaged maize population in the central valley of Oaxaca, México, and implications for improvement. Kluwer Academic Publishers. Netherlands. Euphytica 128: 105-119. https://doi.org/10.1023/A:102064781301

Windhausen, V.S.; Wagener, S.; Magorokosho, C.; Makumbi, D.; Vivek, B.; Piepho, H.-P.; Melchinger, A.E.; Atlin G.N.. 2012. Strategies to Subdivide a Target Population of Environments: Results from the CIMMYT-Led Maize Hybrid Testing Programs in Africa. Crop Sci. 52(5): 2143-2152.

https://doi.org/10.2135/cropsci2012.02.0 
Copyright (c) 2016 C. A. Ramirez Mandujano, J. C. G onzález Cortés,

A. Ávila Bautista y A. A. Hernández Esquivel

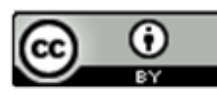

Este tex to está protegido por una licencia licencia Creative Commons 4.0 .

Usted es libre para Compartir —copiar y redistribuir el material en cualquier medio o form ato-y Adaptar el documento —remezclar, transformar y crear a partir del material- para cualquier propósito, incluso para fines comerciales, siempre que cumpla la condición de:

Atribución: Usted debe dar crédito a la obra original de manera adecuada, proporcionar un enlace a la licencia, e indicar si se han realizado cam bios. Puede hacerlo en cualquier form a razonable, pero no de form a tal que sugiera que tiene el apoyo del licenciante o lo recibe por el uso que hace de la obra.

Resumendelicencia - Textocompletodelalicencia 